\title{
The Structure of Tassel in Barren Stalk 1 (ba1) Mutant of Maize
}

\author{
W. C. Cheng ${ }^{1}$, D. B. Walden ${ }^{2}$, R. Alberate ${ }^{3}$ and P. C. Cheng ${ }^{4 *}$ \\ ${ }^{1}$ Dept. of Genetics, University of Wisconsin, Madison, WI, 53703 USA \\ ${ }^{2}$ Dept. of Plant Sciences, Univ. of Western Ontario, London, Ontario, Canada \\ ${ }^{3}$ Dept. of Animal Sciences, University of Wisconsin, Madison, WI, 53703 USA \\ ${ }^{4}$ Division of Bio-engineering., Department of Electrical and Computer Engineering., Department \\ of Radiology, National University of Singapore, Singapore
}

The barren stalk 1 (bal, 3L-94) mutant in maize (Zea mays L.) is characterized by a totally barren tassel inflorescent (Figure 1 and 2). The homozygous bal plant not only develops a male inflorescent totally barren from any branch and spikelet (Figure 2), but also develop no ear shoot (Figure 3). As a result, the plant is essentially male and female sterile. The stock is maintained through the heterozygous (bal/+). We have initiated a study to elucidate the structure of the barren tassel inflorescent. This article reports the morphology of barren tassel stalk at the equivalent stage when wild type at mid silking.

Maize plant were grown and collected at the University of Western Ontario nursery (London, Ontario, Canada) in the summer of 2001 and 2002. Six homozygous (bal/bal) plants were recovered from a total of 40 plants. The specimen was fixed in methanol, cleared in FocusClear and mounted in MountClear (Pacgen Biopharmaceuticals, Vancouver, Canada). Series of optical sections were obtained by using an Olympus Fluoview FV500 confocal microscope operating in epi-fluorescent mode with $488 \mathrm{~nm}$ excitation, $>560 \mathrm{~nm}$ fluorescent was detected. The FocusClear and MountClear made the specimen significantly transparent by reducing scattering, hence allow deeper optical sectioning. For SEM study, fixed specimen was dehydrated in acetone and critical point dried, coated with AuPd and observed with a Hitachi S570 SEM operated at 5kv.

In close inspection, the surface of the barren tassel inflorescent is dotted with small bumps (Figure 2). As revealed by SEM images, the small bumps on the surface of the barren inflorescent are smooth outgrowths with few hairs (Hr) located only at the bottom of each outgrowth (Figure 4; B). Unlike the typical meristematic primodia, the surface of the outgrowth is dotted with stomata (Figure 5; St) and dumbbell-shaped silica cells (Figure 6; Si). In wild type, the tassel is polygonal in cross section; however, the cross section of bal/bal shows a near circular profile (Figure 4). A confocal cross section of the barren inflorescent reveals parallel arranged vascular bundles (Figure 4; V) as found in the wild-type; however, horizontal vascular branching (Figure 4; HV) occurs when approaching the surface outgrowth (Figure 4; B), Hair cells (Hr) are clearly visible in the longitudinal optical section (Figure 7). By analysis the location of the outgrowths on the stalk, it is reasonable to speculate that most of them represent the remnants of aborted spikelets on the now barren tassel stalk. The surface outgrowths at the lower portion of the stalk may represent the remnants of the tassel branches [1].

[1] Cheng et al.,Amer. J. Bot., 70 (1983) 450-460.

* On leave from Department of Electrical engineering, State University of New York at Buffalo

Figure captions: (next page)

FIG 1. Phenotype of bal/bal

FIG 2. Sterile tassel inflorescent of $b a 1 / b a 1$

FIG 3. bal/bal plant with all leave removed showing no ear shoots. 

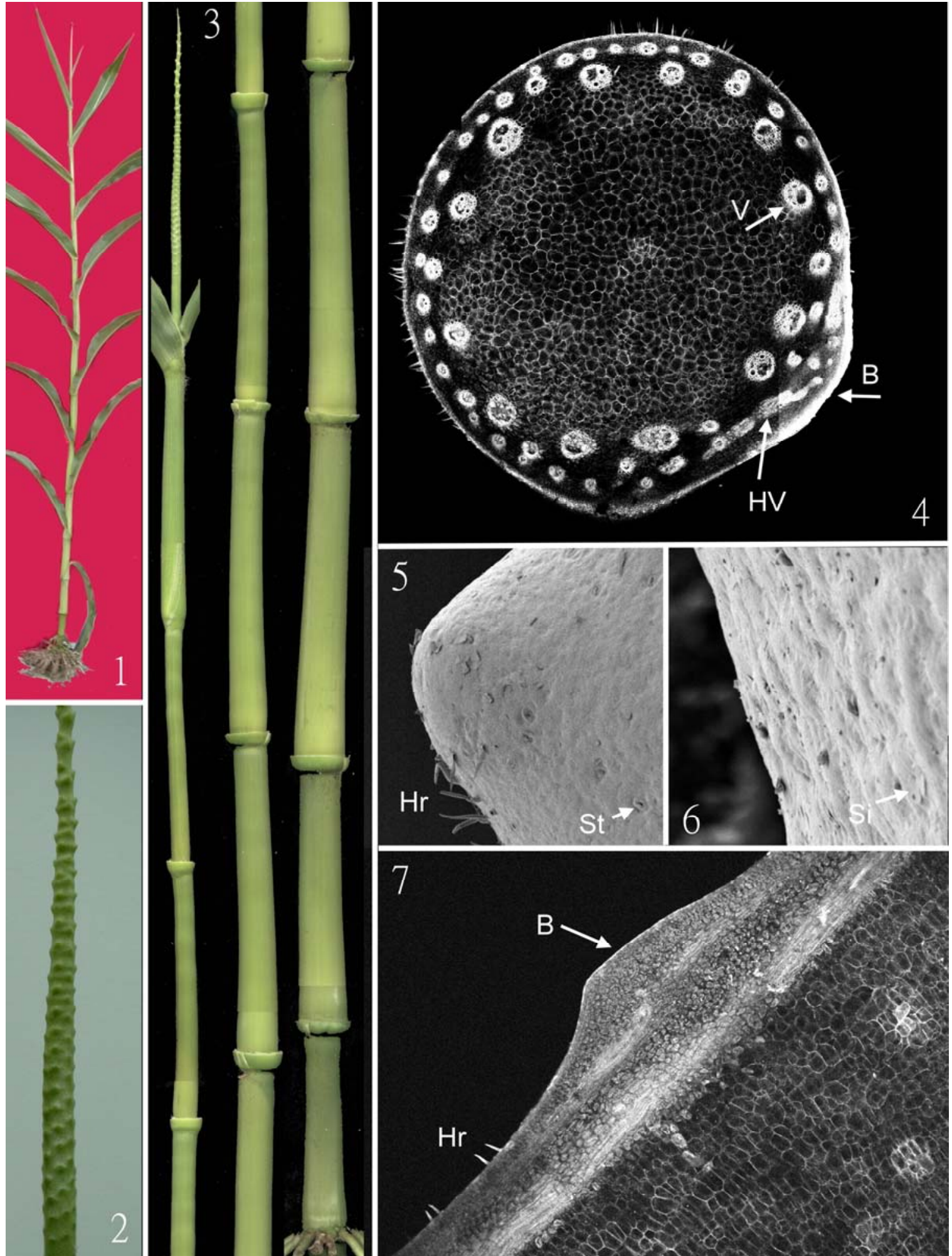

FIG 4. Confocal cross section of ba1/ba1 tassel inflorescent showing vertical vascular bundle (V), horizontal bundle (HV) and surface outgrowth (B).

FIG 5 and 6. SEM image of surface outgrowth. Hr: hair cell, Si: silica cell, St: stomata.

FIG 7. Confocal longitudinal section of ba1/ba1 tassel inflorescent. B: surface outgrowth, Hr: hair cell. 